\title{
An expectorated hydatid
}

\section{Satyajeet Sahoo, Subhra Mitra}

'Department of Pulmonary Medicine and Critical Care, All India Institute of Medical Sciences, Bhubaneswar, Bhubaneswar, India ${ }^{2}$ Calcutta National Medical College and Hospital, Kolkata, India

An 18-year-old housewife presented with a $6 \mathrm{~cm} \times 4 \mathrm{~cm}$ specimen of greenish white material (Figure 1A) which she had expectorated after an episode of violent coughing. She had been suffering from a dry cough for the last 6 months. There was no history of hemoptysis, fever, wheezing, chest pain, loss of weight or appetite. Chest radiograph showed a well circumscribed opacity in the right lower zone and another ill-defined opacity in the left lower zone. A contrast enhanced computed tomographic (CECT) scan of the thorax demonstrated a well-defined, multiloculated lesion with enhancing septation in the right lower lobe abutting the lateral chest wall measuring $5.7 \mathrm{~cm} \times 4.8 \mathrm{~cm}$ in size. A patchy, wedge shaped area with patent bronchograms was also noted in the basal segment of the left lower lobe. There was no evidence of mediastinal lymph node enlargement. An incidental finding of a well-defined multi-loculated cystic lesion in the right lobe of the liver measuring $7.3 \mathrm{~cm} \times 6.3 \mathrm{~cm}$ was also evident (Figure 1B, 1C). Histopathological examination of surgically excised specimens confirmed the diagnosis of pulmonary and liver hydatid cysts.

Hydatid disease is caused by the tapeworms Echinococcus granulosus and Echinococcus multilocularis (alveolaris). The ova passed in feces by the primary host (dogs) are sometimes ingested by humans (intermediate host). Larvae develop in the duodenum of the intermediate host, where they enter the bloodstream and travel to the liver and lungs and occasionally, even the systemic circulation. Pleural or pulmonary involvement may also occur due to direct extension through the diaphragm from hydatid disease in the liver. Disease in humans occurs as a result of cyst formation around the parasite. Unruptured cysts do not usually give rise to symptoms, therefore, the diagnosis is based on a routine chest film. Approximately two-thirds of pulmonary hydatid cysts rupture. Most rupture into the surrounding lung and bronchial tree, causing secondary infection. Occasionally, a cyst ruptures into the pleural cavity. Rupture may result in an acute allergic reaction, sometimes accompanied by life-threatening hypotension. In pulmonary hydatid cysts, the cardinal imaging features are one or more spherical or oval well-defined smooth masses of homogeneous density in otherwise normal lung tissue, usually with a predilection for the lower lobes, the posterior segments and the right lung. Multiple cysts are seen in about a third of patients and are bilateral in $20 \%$ [1, 2]. In cases of pulmonary hydatid cysts, surgery is the treatment of choice.
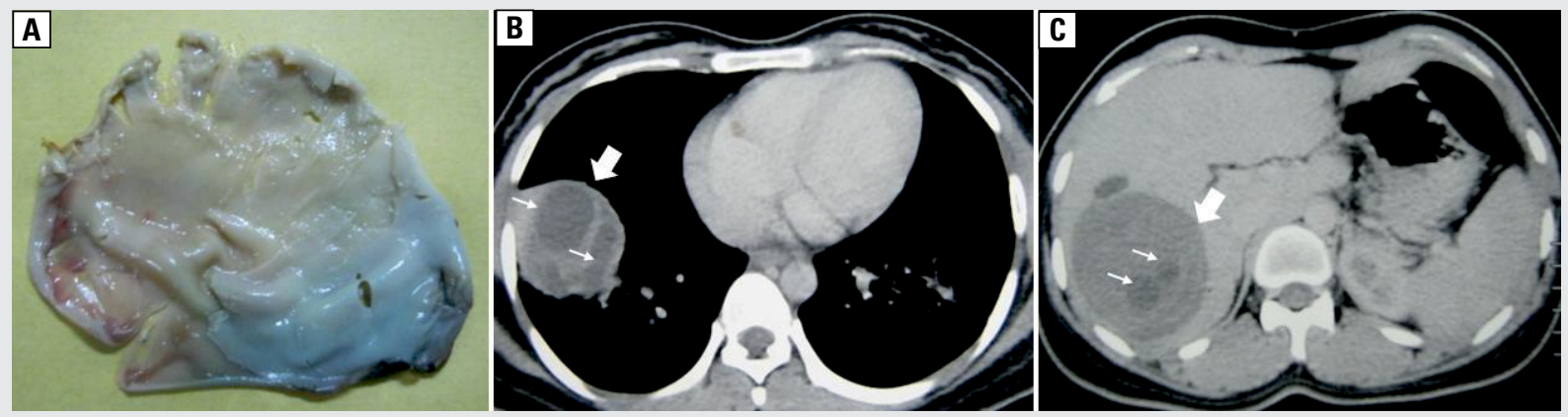

Figure 1. A. Expectorated greenish white material. B. Axial section of CECT scan of chest showing a well-defined lesion of $5.7 \mathrm{~cm} \times 4.8$ $\mathrm{cm}$ size (block arrow) with enhancing septation in right lower lobe abutting the chest wall laterally with daughter cysts (arrows). C. Another well-defined cystic lesion in right lobe of liver measuring $7.3 \mathrm{~cm} \times 6.3 \mathrm{~cm}$ (block arrow) with presence of daughter cysts within (arrows)

\section{References:}

1. Kervancioglu R, Bayram M, Elbeyli L. CT findings in pulmonary hydatid disease. Acta Radiol. 1999; 40(5): 510-514, indexed in Pubmed: 10485240 .

2. Erdem CZ, Erdem LO. Radiological characteristics of pulmonary hydatid disease in children: less common radiological appearances. Eur J Radiol. 2003; 45(2): 123-128, doi: 10.1016/s0720-048x(02)00054-2, indexed in Pubmed: 12536091.

Address for correspondence: Satyajeet Sahoo, Department of Pulmonary Medicine and Critical Care, AllMS, Sijua, Patrapada, Bhubaneswar, Odisha, India; e-mail: satyajeetsahoo@yahoo.co.in

DOI: 10.5603/ARM.2019.0055

Received: 14.08 .2019

Copyright (C) 2019 PTChP

ISSN 2451-4934

Conflict of interest: none declared 